\title{
Research on Recognition Algorithm of Track Substructure Defects Based on Vehicle Dynamic Responses
}

\author{
Hongmei Shi \\ School of Mechanical \& Electronic Control Engineering, Beijing Jiaotong Univerisity \\ 100044 Beijing, China \\ hmshi@bjtu.edu.cn
}

\begin{abstract}
Track stiffness abrupt change caused by track substructure defects can lead to rather large dynamic irregularity when the train goes through it. Thus the dynamic action between wheel and rail is increasing, which will influence the safety and comfort of the train. In this paper, the dynamic responses of vehicle and track are analyzed based on vehicle track coupling model in which two kinds of defects of track substructure are simulated. To reduce the maintenance cost, a recognition algorithm of track substructure defects using vehicle dynamic responses is proposed based on Support Vector Machines. Finally, a case has been studied based on the algorithm proposed in this paper, and the simulation results show that the method is effective to recognize the track substructure defects such as unsupported sleepers, loosened fasteners, and the accuracy of recognition can be reached to $90 \%$.
\end{abstract}

Keywords: track substructure defects, vehicle track coupling model, vehicle dynamic responses, support vector machine, reduce cost.

\section{$1 \quad$ Introduction}

When the defects exist in the track substructure, such as unsupported sleepers, loose fasteners, the track stiffness will vary largely within a short distance which is called track stiffness irregularity and it leads to the increased dynamic action between train and track. The rail vibration is obviously aggravated and the interaction force come a large transition to cause an impulse due to the track substructure defects, which will influence the train safety and ride comfort meanwhile it will intensify track degradation because worse track geometry irregularities will be brought. Hence, it is very important to inspect the track substructure defects in time to ensure the safety operation of train. Now the track defects are often detected visually by maintenance staff or periodically by special inspection vehicle. All these methods are timeconsuming and have potential danger if the defects cannot be found in time between the inspection periods. A detection method is proposed in this paper, which the sensors are mounted on the normal trains in service and the vehicle dynamic responses are used to detect the track defects based on the proposed algorithm in this paper. 
In recent years, a few scholars have investigated the vehicle track dynamic responses considering the track stiffness change. Luo etc. [1-2] studied the influence of the track stiffness on the vehicle and rail deformation at high-speed railway bridge transition regions. Sun and Chen etc. [4-5] investigated the distribution rules of the track stiffness at the turnout regions and the reasonable value for the ballastless track. Zhu and Ahmed etc. [6] made a research on the dynamic interaction relationship between wheel and rail under the condition of the unsupported sleepers with vehicletrack spacial model and the gap size influence on the interaction force. Lei [7] studied the influences of four transition patters including abrupt change, step change, linear change and cosine change on the vehicle and track dynamic responses at the speedup railway. The research results show that the cosine change of the track stiffness is the most effective to the train operation safety at the transition regions.

In this paper we are aimed at solving the problem of the track substructure defects recognition based on the vehicle dynamic responses, thus we can mount the accelerators on the in-service vehicle to find the track substructure defects, no need to arrange the special inspection vehicle [8] to run just for the defects recognition which is costly and need a long occupied period. The paper is organized as follows: Section 2 introduces the vehicle-track coupling model proposed by Zhai and Sun, which is used in the Section 3. Section 3 simulated and analyzed the dynamic vehicle and track responses under the condition of track substructure defects based on the model introduced in Section 2. Section 4 proposed a recognition algorithm with vehicle dynamic responses based on Support Vector Machine, in which genetic algorithm and PSO algorithm are separately used to optimize the SVM parameter to get the better recognition results. And a case is studied based on this proposed method, and the results show that the algorithm proposed in this paper is effective to track substructure defects recognition.

\section{Vehicle- Track Vertical Coupling Model}

Zhai and Sun vehicle - track vertical nonlinear coupling model [9] is adopted in this paper, consisting of vehicle model and track model as shown in Fig. 1. The vehicle is viewed as a multibody system running on the rail at the speed of $v$, consisted of the car body, the front and rear bogies, the wheelsets. $M_{\mathrm{c}}$ is mass of the car body and $J_{\mathrm{c}}$ is its nod moment of inertia, and $M_{\mathrm{t}}$ is mass of the bogie and $J_{\mathrm{t}}$ is its nod moment of inertia. $M_{\mathrm{w}}$ is mass of each wheelset. $K_{\mathrm{pz}}$ is the stiffness coefficient and $C_{\mathrm{pz}}$ is the damping coefficient of the primary suspension. $K_{\mathrm{sz}}$ is the stiffness coefficient and $C_{\mathrm{sz}}$ is damping coefficient of the second suspension system. Track model uses rail-sleeper-ballast-subgrade three layers model. Rail is viewed as an infinite length Euler beam resting on continuous elastic support which is discretized along the longitude. Rail sleeper uses rigid unit and ballast is discretized into block unit. 


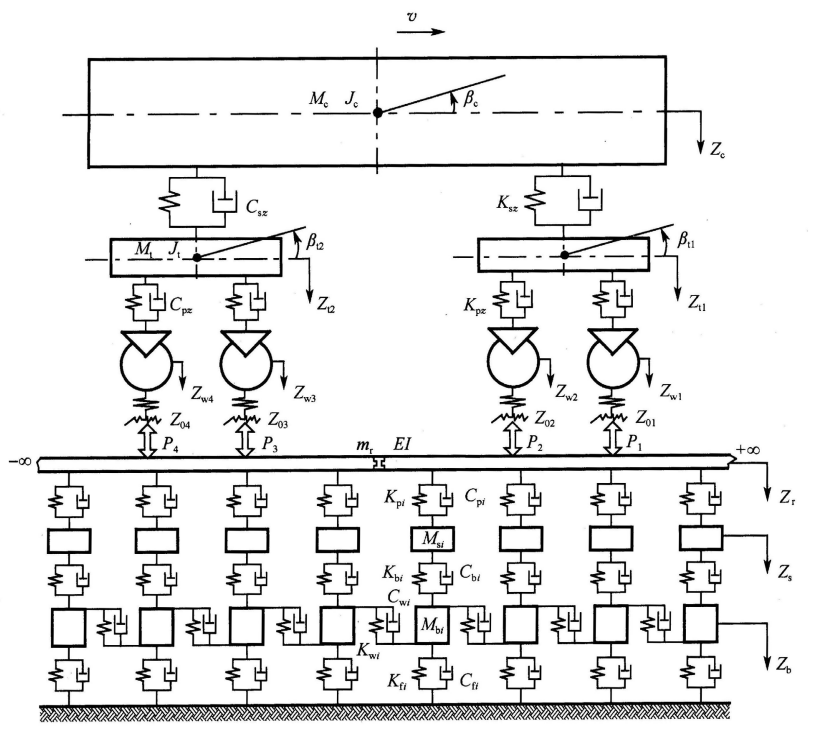

Fig. 1. Vehicle track vertical coupling model

The vertical coupling relationship is realized by vertical wheel and rail contact model, expressed in the equation of vertical interaction force. In this paper Hertz nonlinear elastic contact model is used, which is also the most classic and effective model.

\section{Vehicle Track Dynamic Responses under the Condition of the Track Substructure Defects}

Generally, the track substructure defects include unsupported sleeper, loosened rail fasteners, loose or hardened ballast. The first two kinds of defects occasionally exist after long-time train operation, which are also mainly discussed in this paper. Here we adopt the different value for the different track layer stiffness and damping to simulate the track substructure defects. For a loosened fastener, we suppose in the Fig. 1 model $K_{p i}=C_{p i}=0$. For an unsupported sleeper, we suppose in the Fig. 1 model $K_{b i}=C_{b i}=0$.

\subsection{Loosened Fasteners Condition}

First we suppose there are 5 loosened fasteners along 100m track, which are No. 40, No.70, No. 110, No. 130 and No. 160 (which is selected randomly), to calculate the vehicle and track dynamic responses under this situation. The other simulation conditions are as following: the train speed is $160 \mathrm{~km} / \mathrm{h}$, the track condition adopts China speedup line parameter. The calculation software is coded with Matlab, in which the vehicle and track dynamic responses are calculated by numerical integral method. The simulation results is shown in Fig. 2 which is expressed in the frequency domain, and the red curve represents the dynamic responses with the defect of loosened fasteners, the blue one is the normal situation without defects. 


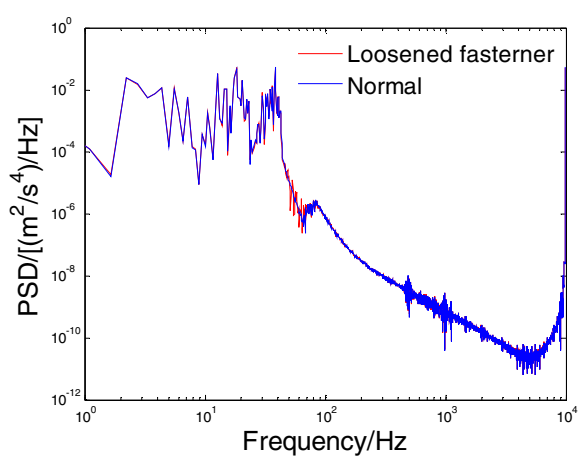

(a) carbody vibration acceleration PSD

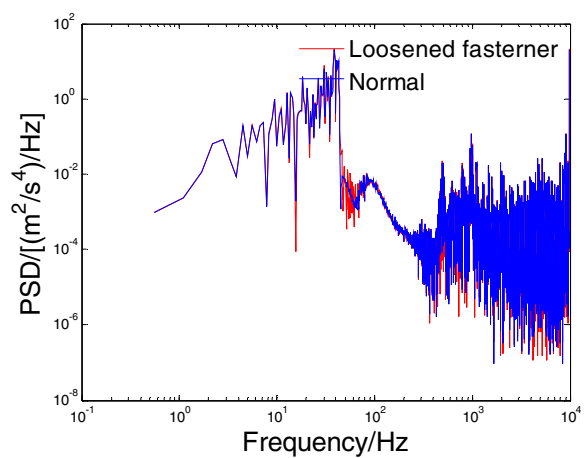

(c) wheelsets vibration acceleration PSD

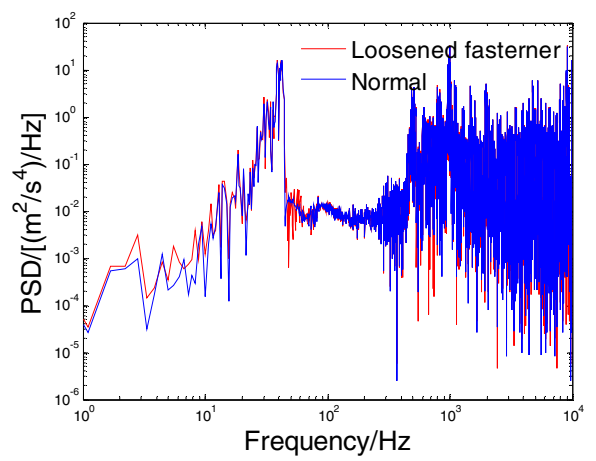

(e) rail vibration acceleration PSD

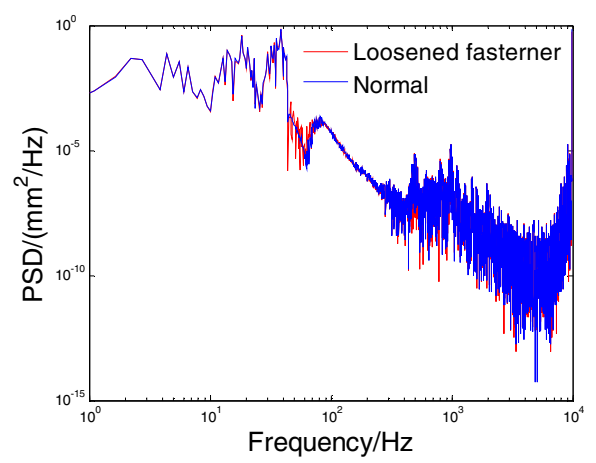

(b) vehicle bogie vibration acceleration PSD

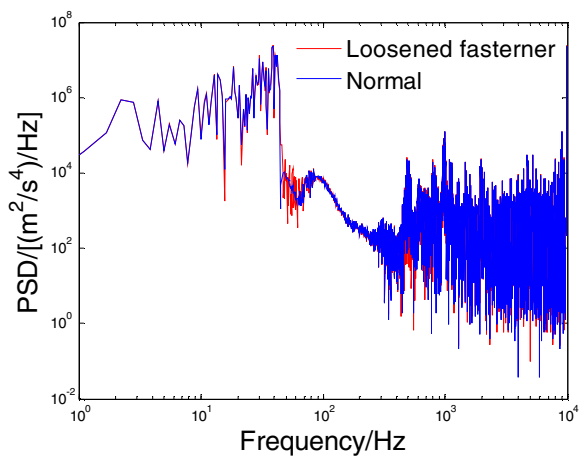

(d) interaction force PSD

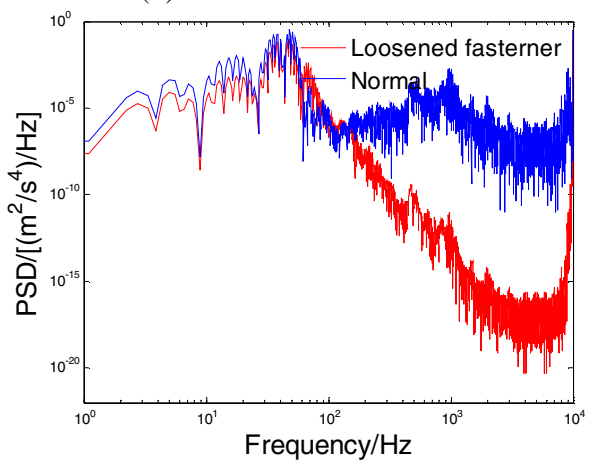

(f) No.70 sleeper vibration acceleration PSD

Fig. 2. Vehicle and track dynamic responses under the condition of loosened fasteners

The difference can be seen from Fig.2 between normal situation and defects condition at the $40 \mathrm{~Hz}$ to $80 \mathrm{~Hz}$, but the difference on vehicle dynamic responses is minor while the track is more obvious. The vehicle dynamic responses are at low frequency and track dynamic responses are at higher frequency. At the same time, the vertical displacement of the track components are compared and it is found that rail vertical displacement is increased obviously but those of sleeper and ballast are decreased. 


\subsection{Unsupported Sleeper Condition}

Then we suppose there are 5 unsupported sleepers along $100 \mathrm{~m}$ track, which are No. 40, No.70, No. 110, No. 130 and No. 160, to calculate the vehicle and track dynamic responses under this situation (in order to compare the different defects influences, we

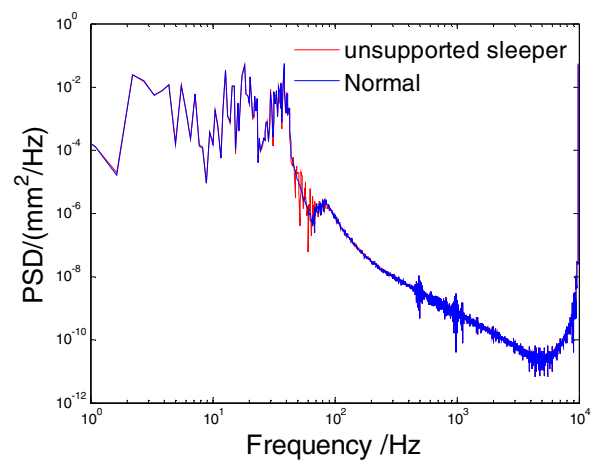

(a) carbody vibration acceleration PSD

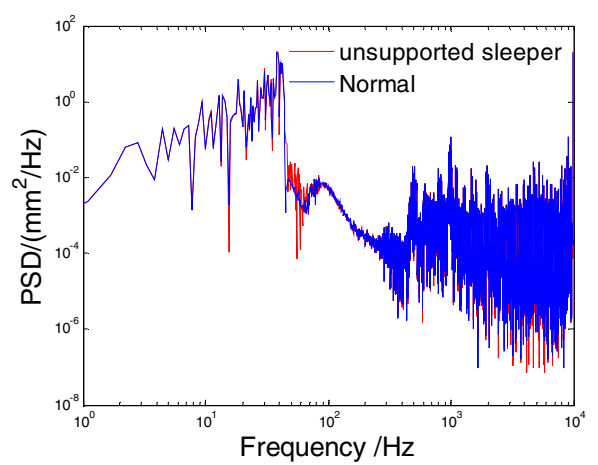

(c) wheelsets vibration acceleration PSD

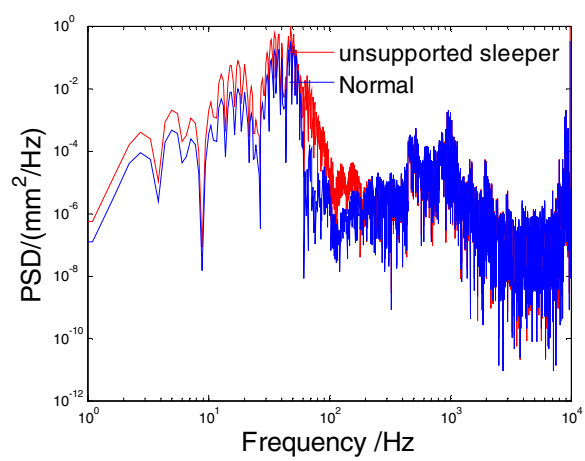

(e) No.70 sleeper vibration acceleration PSD

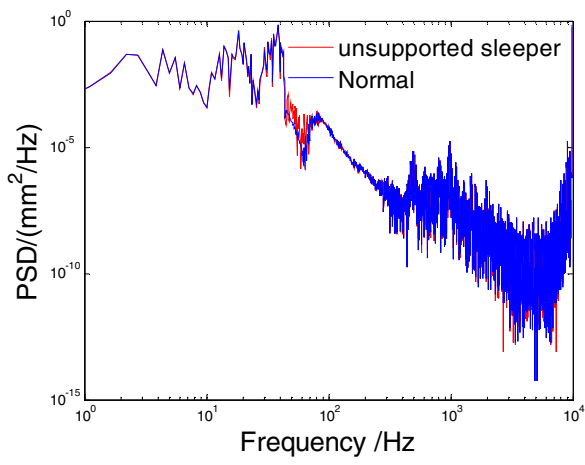

(b) vehicle bogie vibration acceleration PSD

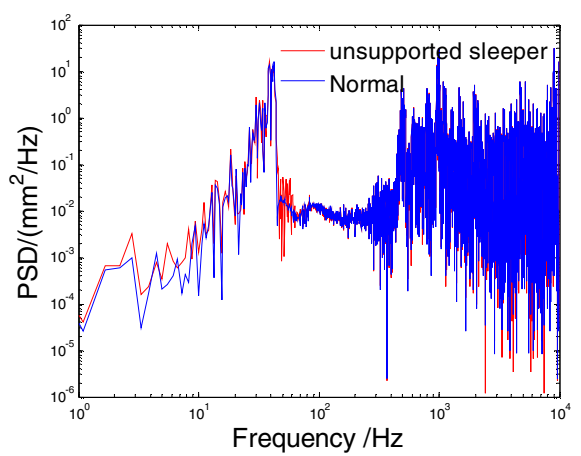

(d) rail vibration acceleration PSD

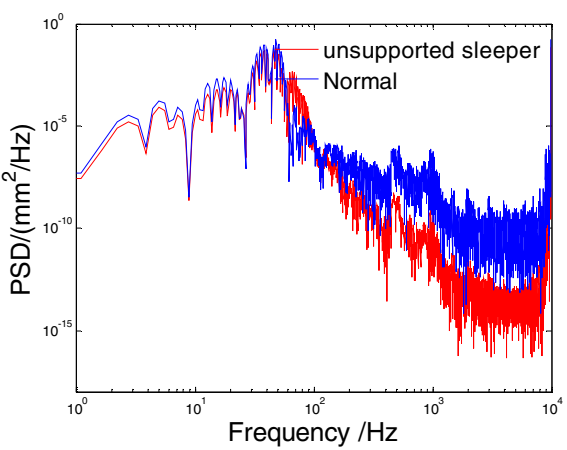

(f) No. 70 ballast piece accelertion PSD

Fig. 3. Vehicle and track dynamic responses under the condition of unsupported sleeper 
define the same defect positions). The other simulation conditions are the same as previous simulation. The simulation results under the condition of unsupported sleepers are shown in Fig. 3 which is also expressed in the frequency domain, and the red curve represents the dynamic responses with the defect of unsupported sleepers, the blue one is the normal situation without defects.

The difference can be seen from Fig. 3 between normal situation and defects condition, the same as loosened fastener situation, the difference on vehicle dynamic responses is minor while the track is more obvious. At the same time, the vertical displacement of the track components are also compared and it is found that rail and sleeper vertical displacement are increased obviously but ballast displacement is decreased. It also can be found that vehicle dynamic responses vary unobviously under the different track substructure defects conditions.

\section{Recognition Algorithm of Track Substructure Defects Based on Support Vector Machine}

Based on the above simulation results, we can easily come to the conclusion that the track dynamic responses are more obvious than the vehicle under the condition of track substructure defects. For example, it can be recognized easily by the rail displacement. So theoretically, we can recognize the defects according to the track dynamic responses with simple algorithm. But there are thousands of miles railway lines in China, it is impossible to mount sensors along all the in-service lines for the cost and maintenance work. Hence, a recognition algorithm is proposed in this paper, which makes use of vehicle dynamic responses and Support Vector Machine (SVM).

The vehicle track coupling model in Section 2 is nonlinear model due to the wheelrail contact relationship, so it is also impossible to solve the track stiffness parameters by the vehicle dynamic responses. Support Vector Machine is a newly developed pattern recognition method in recent years, which foundation is statistical learning theory and structural risk minimization. SVM uses a nonlinear Kernel function to transform input data to a high-dimensional feature space in which the input data become linearly separated.The training sample vehicle responses data come from the results of Section 3, and there are three kinds of data, one is wheelsets vibration acceleration under the normal condition (i.e., no defects in track substructure), the second is wheelsets vibration acceleration under the condition of loosened fasteners, and the third is wheelsets vibration acceleration under the condition of unsupported fasteners. The total sample data is 123 groups, 90 as training sample and 33 as testing sample. There are 11 normal condition, 11 unsupported sleepers, 11 loosened fasteners in the testing sample data. Each group has 368 data.

The selection of kernel function of SVM is an important step, the popular admissible kernels include Gaussian RBF, polynomial, Sigmoid etc. Gaussian RBF is chosen as kernel function in this paper. There are two learning parameters in constructing SV machines, i.e., the penalty parameter $\mathrm{C}$ (that determines the trade-off between the training error and VC dimension of the model), and the shape parameters of the kernel function (variances of a Gaussian kernel). The most common method to 
choose the parameters is a cross-validation. To improve the SVM classification effective, we also adopt genetic algorithm and PSO algorithm to optimize the $C$ and $\delta$. Table 1 is the comparison results with different parameter selection methods. The SVM classification procedure of track defects is coded with Matlab. The input data of SVM are wheelset acceleration and the output is number $0,1,2$ which respectively express normal, loosened fastener, and unsupported sleeper.

Table 1. SVM Performance comparison for each parameter selection methods

\begin{tabular}{cccc}
\hline & Cross-validation & PSO & GA \\
\hline Classification & $90.91 \%$ & $100 \%$ & $96.97 \%$ \\
Accuracy Rate & 331.34 & 211.72 & 25.04 \\
Calculation time (s) & $\mathrm{C}=1$ & $\mathrm{C}=31.1735$ & $\mathrm{C}=82.2372$ \\
Parameter Value & $\delta=32768$ & $\boldsymbol{\delta}=420.2098$ & $\boldsymbol{\delta}=68.4522$ \\
\hline
\end{tabular}

For the cross-validation method, if we want to get the optimum value, the search range must be large and step must be small, but this will cause a longer calculation time, or the accuracy can't be achieved. PSO algorithm can get a higher accuracy but the calculation time is longer than GA.

Then we use the recognition algorithm to study a supposed case to test the algorithm validity. Suppose that there are six track substructure defects along $100 \mathrm{~m}$ long track as follows, No.61, No. 62, No. 63, No. 100 fasteners are loosened, No.130 and No.160 sleepers are unsupported(the samples are also selected randomly to show the adaptability of algorithm). The vehicle axle-box accelerations (wheelset acceleration) are used to identify the track substructure defects. The recognition results are shown in Table 2.

Table 2. Recognition results of track defects

\begin{tabular}{|c|c|c|}
\hline $\begin{array}{l}\text { Track } \\
\text { status }\end{array}$ & The true situation & Recognition results \\
\hline Normal & 136 positions & 126 positions \\
\hline $\begin{array}{l}\text { Loosened } \\
\text { fastener }\end{array}$ & $4(61,62,63,100)$ & $10(60, \underline{61,62,63,64,65,100,103,127, \underline{130})}$ \\
\hline $\begin{array}{l}\text { Unsupported } \\
\text { sleeper }\end{array}$ & $2(130,160)$ & $6(97,99,131,132, \underline{160,161)}$ \\
\hline
\end{tabular}

The classification accuracy rate is $92.25 \%$ calculated from Tab. 2. The wrong recognition positions are all around the true defects, and all the track defects have been recognized, but one position is classified wrongly (No. 130). In fact, this case is a rather worse situation because there are several continuous loosened fasteners which lead to the influence on vehicle dynamic responses at the near defects track position. This situation is not common in the real, and the wrong recognized positions are all around the defects which can be acceptable by maintenance. 


\section{Conclusion}

Track substructure defects are one of the reasons leading to track stiffness irregularity, which is one of the dynamic excitation sources to the vehicle track coupling system,. In this paper a recognition method of track substructure defects is proposed base on vehicle track coupling model and support vector machine. With the SVM advantages, which can realize the linear classification in high-dimension space using kernel function, the track substructure defect recognition and classification has been achieved based on vehicle dynamic responses. This method can provide a new idea for the track condition inspection from the in-service vehicles.

Acknowledgments. This paper is supported by the National Natural Science Foundation of China (60972092 \& 61134003) and the Fundamental Research Funds for the Central Universities (2012JBM094).

\section{References}

1. Qiang, L., Ying, C., Wanming, Z.: Dynamic Performance Analysis of High-speed Railway Bridge-approach Embankment. Engineering Mechanics 16, 65-70 (1999)

2. Pingrui, Z., Likang, G.: Bridge Transition Section Stiffness and Deformation of Ballastless Track. Subgrade Engineering 4, 18-20 (2009)

3. Ping, W., Xueyi, L., Zhonghou, K.: Study on Turnout Vertical Stiffness Distribution Rules Along the Line. Journal of Southwest Jiaotong Univerisity 34, 144-147 (1999)

4. Xiaoping, C., Ping, W.: Distribution Regularity and Homogenization of Track Rigidity for Ballastless Turnout. Journal of Southwest Jiaotong Univerisity 41, 447-451 (2006)

5. Xiaoping, C., Ping, W.: Research on Reasonable Stiffness of Ballastless Turnout for 350km/h Passenger-Dedicated Line. Railway Standard Design 3, 1-3 (2010)

6. Jian Jun, Z., Ahmed, A.K.W.: Development of a Vehicle-track Model Assembly and Numerical Method for Simulation of Wheel-rail Dynamic Interaction due to Unsupported Sleepers. Vehicle System Dynamics 48, 1535-1552 (2010)

7. Xiaoyan, L., Lijun, M.: Dynamic Response Analyses of Vehicle and Track Coupled System on Track Transition of Conventional High Speed Railway. Journal of Sound and Vibration 271, 1133-1146 (2004)

8. Eric, G.B., Amir, M.K., Bjorn, D.: Identification Properties of Railway Tracks by Dynamic Stiffness Measurements and Simulation. Journal of Sound and Vibration 329, 3999-4016 (2010)

9. Wanming, Z.: Vehicle-Track Coupling Dynamics. Science Press, Beijing (2007) 